\title{
Alterstice
}

Revue internationale de la recherche interculturelle

International Journal of Intercultural Research

Revista International de la Investigacion Intercultural

\section{La diversité culturelle dans les PME, accès au travail et valorisation des ressources, sous la direction de A. Manço et}

\section{Barras}

\section{Jamal-Eddine Tadlaoui}

Volume 4, numéro 1, 2014

URI : https://id.erudit.org/iderudit/1077485ar

DOI : https://doi.org/10.7202/1077485ar

Aller au sommaire du numéro

Éditeur(s)

Alterstice

ISSN

1923-919X (numérique)

Découvrir la revue

Citer ce compte rendu

Tadlaoui, J.-E. (2014). Compte rendu de [La diversité culturelle dans les PME, accès au travail et valorisation des ressources, sous la direction de A. Manço et

C. Barras]. Alterstice, 4(1), 95-97. https://doi.org/10.7202/1077485ar d'utilisation que vous pouvez consulter en ligne. 


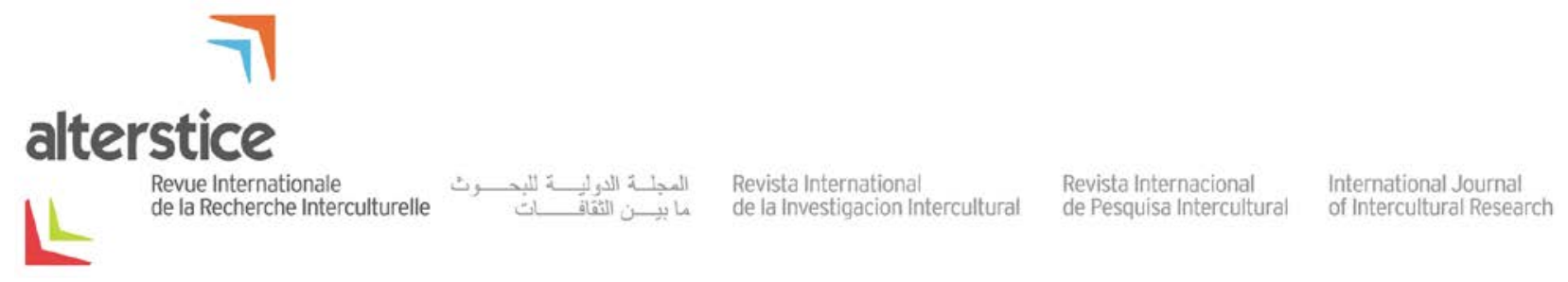

NOTE DE LECTURE

\title{
La diversité culturelle dans les PME, accès au travail et valorisation des ressources, sous la direction de A. Manço et C. Barras
}

Jamal-Eddine Tadlaoui $^{1}$

\author{
Rattachement de l'auteur \\ ${ }^{1}$ Université de Sherbrooke, Sherbrooke (Québec), Canada
}

\section{Correspondance}

jamal-eddine.tadlaoui@usherbrooke.ca

\section{Références de l'ouvrage}

Manço, A. et Barras, C. (dir.) (2013). La diversité culturelle dans les PME, accès au travail et valorisation des ressources. Paris : L'Harmattan.

\section{Pour citer cet article}

Tadlaoui, J.-E. (2014). La diversité culturelle dans les PME, accès au travail et valorisation des ressources [Note de lecture]. Alterstice, 4(1), 95-98.

\begin{abstract}
Les réflexions et les débats sur la diversité culturelle et sa gestion au sein des entreprises ne datent pas d'aujourd'hui, notamment en Europe, au Canada et au Québec, historiquement terres d'accueil. Plusieurs chercheurs s'y sont investis, en empruntant diverses avenues théoriques tant pour traiter des différents types de diversité culturelle en entreprise et établir des comparaisons entre plusieurs contextes de travail que pour suggérer des pistes d'action et de réflexion à propos des stratégies gagnantes de gestion de la diversité culturelle dans des milieux de travail de plus en plus diversifiés. Pour leur part, Altay Manço et Christine Barras, directeurs de ce « livre diversifié ", publication de l'Institut de recherche, formation et action sur les migrations (IRFAM) ${ }^{1}$, nous offrent une contribution collective intéressante et originale. Ils signent également personnellement quelques articles dans l'ouvrage. Par sa structure, ses thèmes, la nature des analyses et les profils personnels et professionnels des 23 auteurs œuvrant dans cinq pays européens et au Québec, cette publication va certainement alimenter la réflexion autour de la gestion de la diversité culturelle au sein des petites et moyennes entreprises (PME) tout en valorisant l'apport et la plus-value professionnelle de cette diversité, sous-estimée voire méconnue par certains chefs de PME.
\end{abstract}

\footnotetext{
${ }^{1}$ Voir la présentation complète de l'IRFAM p. 376 de l'ouvrage.
} 
Soutenue par des assises théoriques, largement inspirées des travaux de Minzberg quant à la gestion des contraintes imposées à l'organisme par l'environnement socioculturel, et par les résultats des travaux de Pichault et Nizet (1995-2000), l'une des caractéristiques principales de cette publication réside dans « la mise en valeur de stratégies de gestion de la diversité ethnoculturelle dans les entreprises au bénéfice du bien-être au travail " (p. 15). Les résultats qui découlent des différentes contributions vont se traduire par une mise en commun et un partage des meilleures pratiques de gestion de la diversité culturelle, tant avec les services des ressources humaines des PME qu'avec d'autres intervenants responsables des bassins de main-d'œuvre diversifiée. D'ailleurs, les directeurs de publication précisent que les nouvelles pratiques, savoirs-faire et savoirs-être décrits dans ces contributions feront l'objet d'un programme de formation continue pour les intervenants agissant dans le champ de la gestion de la diversité culturelle au sein de milieux de travail variés.

Sur près de 400 pages, cet ouvrage collectif présente les résultats de recherches exploratoires issues d'analyses qualitatives (certaines plus poussées que d'autres) et soumises à un processus de recherche scientifique encadré et coordonné par les directeurs de publication. Il est composé de quatre grandes parties, dont la deuxième est plus fournie que les autres. Ces parties vont de l'accès au travail des nouveaux arrivants à une analyse transversale (une sorte de retour analytique-conclusion-recommandations sur l'ensemble des contributions) en passant par des expériences de gestion de la diversité en entreprise et des actions et pratiques efficaces en vue de mieux y valoriser la diversité culturelle ou interculturelle. Nous ne désirons pas ici revenir en détail sur ces différentes parties différemment illustrées, mais nous voudrions plutôt nous concenter sur l'une d'elles, très originale, celle de l'analyse transversale menée par les directeurs de publication de l'ouvrage. Du fait de l'approche méthodologique conjointe de A. Manço et C. Barras et des autres auteurs quant à l'ensemble du processus de recherche, d'analyse et de présentation des résultats, la partie relative à l'analyse transversale a toute sa légitimité, sa pertinence et sa portée pédagogique, tant pour les intervenants que pour les gestionnaires des entreprises, notamment les petites et moyennes. À partir d'une "méta-analyse », les directeurs de publication reviennent abondamment sur les différents articless de l'ouvrage, au moyen d'observations systématisées et d'un retour méticuleux sur les 20 monographies regroupées en trois parties. Ce retour analytique s'est fait à partir des sept dimensions clés qu'ils avaient ciblées et que les auteurs des articles ont utilisées dans leurs développements respectifs. Directement reliées à la thématique de la gestion de la diversité culturelle ou interculturelle en entreprise et adaptée aux divers contextes des contributions variées de l'ouvrage, ces dimensions sont intitulées recrutement, socialisation, formation, équipes, carrière, accommodements et recommandations. Elles ont été analysées et comparées entre elles en fonction de la pertinence des propos des auteurs respectifs et de leur utilisation appropriée ou non dans la pratique de la gestion de la diversité en entreprise.

Plutôt que de s'arrêter sur l'analyse de chacune des dimensions, ce qu'ont fait systématiquement A. Manço et C. Barras, rappelons les conclusions qui en découlent, en parallèle à la question du pouvoir en entreprise qu'ils ont voulu mettre en perspective. Cette relation découle du fait que le pouvoir en entreprise est « lié à la dynamique du changement à l'ouverture et à la prise de risque » (p. 357). Ici, le changement correspond au changement social. Autrement dit, à travers l'analyse des sept dimensions en question, les directeurs de publication invitent à évaluer et à mesurer les pratiques de gestion de la diversité culturelle ou interculturelle en entreprise en lien avec les différentes facettes du pouvoir qui y sont appliquées. En effet, une pratique efficace et l'application à bon escient de cette gestion sont tributaires de ce pouvoir et de ceux qui en usent, tant les patrons que les employés euxmêmes. Ainsi, tant l'identité en son sens le plus large (et notamment l'identité professionnelle) et l'intégration socioéconomique et politique des migrants que les éléments reliés aux différents types de discrimination, l'équilibre social et le bien vivre ensemble dépendent des façons d'exercer le pouvoir, aussi bien dans les entreprises qu'au sein de la société.

Grâce à cette analyse transversale, A. Manço et C. Barras mettent en valeur les conclusions et recommandations des auteurs, qui réaffirment et valorisent d'une façon originale la place et le rôle de la diversité sous toutes ses formes dans une société, sans pour autant l'enfermer dans une lecture essentialisante. Ils proposent une mise en valeur des meilleures pratiques de gestion de la diversité interculturelle, notamment dans les entreprises qui, pour demeurer vivantes et performantes, n'ont plus le choix que d'être de plus en plus diversifiées, mondialisation oblige!

Alterstice - Revue Internationale de la Recherche Interculturelle, vol. 4, $n^{\circ} 1$ 
Dans son ensemble, La diversité culturelle dans les $P M E$, accès au travail et valorisation des ressources est un ouvrage collectif original et diversifié, suffisamment documenté sur les sujets de l'interculturel et de la gestion de la diversité en entreprise. Non seulement il vient alimenter la réflexion sur ce grand thème de l'heure, mais il offre une somme d'informations multidimensionnelles fort utiles et inspirantes. Utilisé notamment par des gestionnaires en entreprise, des professionnels ou des futurs professionnels dans le domaine de l'interculturel, cet ouvrage ne peut qu'ouvrir de nouvelles perspectives d'analyse.

Cela dit - ce qui n'enlève rien aux contributions des auteurs et à l'intérêt qu'il y a à scruter ce qui se passe au sein des PME quant à leur appropriation-réticence, utilisation-rejet de la diversité culturelle -, il est important de rappeler certains éléments complémentaires qui pourraient profiter aux lecteurs. À cet effet, nous nous permettons de signaler que le traitement de la gestion de la diversité culturelle ou interculturelle en entreprise pourrait s'inspirer d'une proposition novatrice plus large. Il s'agit de l'interculturalisme à la québécoise dépeint par le sociologue Gérard Bouchard (2012) dans son dernier essai comme le modèle par excellence, la voie d'avenir pour le Québec quant à sa gestion de la diversité ethnoculturelle, y compris dans les entreprises ${ }^{2}$.

En parallèle à cette première suggestion, nous voudrions ajouter un autre élément, aussi important et peu abordé dans l'ouvrage collectif : les médiations interculturelles et leur rôle quant à la gestion de la diversité en entreprise. En effet, si l'esprit et la philosophie fondamentale des médiations interculturelles et de leurs stratégies étaient mieux considérés, plus valorisés et éthiquement appliqués, notamment dans les milieux de travail, celles-ci permettraient aussi de tendre vers l'équilibre et l'idéal recherchés, y compris en milieu de travail.

Quoi qu'il en soit, l'intégration et l'application effective dans les entreprises comme dans nos vies de l'esprit et de la philosophie positive de la gestion de la diversité et de ses orientations particulières aussi bien que des innombrables mécanismes de médiations interculturelles ne peuvent que faire avancer nos sociétés démocratiques vers plus d'harmonie, de respect mutuel, de justice sociale, en somme vers une véritable reconnaissance de l'Autre, avec moins de domination sous toutes ses formes. Construire ou rebâtir une société égalitaire fondée sur et pour la reconnaissance effective de la diversité se fait en repensant ces nouvelles réalités à partir d'une réelle inclusion et de décisions justes, loin d'une quelconque subordination, y compris celle de certains patrons d'entreprise.

Les directeurs de publication de cet ouvrage collectif conviendraient certainement que des espaces d'amélioration existent et ils poursuivront certainement leurs efforts pour bonifier de prochaines publications sur le sujet. II est en effet légitime d'aspirer à de bons moyens et de bonnes pratiques pour arriver à une meilleure intégration et à une nouvelle gestion de la diversité, et ce, dans des contextes sociétaux, locaux et internationaux qui présentent de nombreux signes de dérèglement et de perturbation de leurs systèmes de valeurs, et cela n'épargne pas les entreprises.

\section{Références}

Bouchard, G. (2012). L'interculturalisme, un point de vue québécois. Montréal : Boréal.

Tadlaoui, J.-E. (2012). L'interculturalisme, un point de vue québécois [note de lecture]. Diversité urbaine, 12(2), 155-157.

\footnotetext{
${ }^{2}$ L'interculturalisme selon Bouchard est également présenté, et avec justesse, comme un modèle à construire dans ses aspects philosophiques et politiques. Compris dans le sens d'un "pluralisme intégrateur ", il devrait s'accomplir au sein de la société québécoise avec toutes ses composantes, c'est-à-dire avec sa majorité et ses minorités, et ce, avec les prérequis de la démocratie et du respect réel de ces composantes. Celles-ci comprennent, notamment, les divers consensus, le respect mutuel et le " bien vivre ensemble " pour tous les citoyens, sans aucune distinction, ni ethnique, ni culturelle, ni religieuse. Au centre du modèle proposé, la "laïcité inclusive » et la dualité majorité/minorités sont deux concepts clés, largement développés tout au long des chapitres de l'essai. Voir notre compte-rendu de cet essai (Tadlaoui, 2012) dans Diversité urbaine.
} 